Astron. Astrophys. 146, 76-80 (1985)

ASTRONOMY

AND

ASTROPHYSICS

\title{
Polycyclic aromatic hydrocarbons and the diffuse interstellar bands
}

\author{
G. P. van der Zwet $^{1}$ and L. J. Allamandola ${ }^{2}$ \\ 1 Laboratorium Astrofysica, Leiden University, Wassenaarseweg 78, Leiden, The Netherlands \\ ${ }^{2}$ National Research Council Senior Associate, NASA Ames Research Center, M.S. 245-6, Moffett Field, CA 94035, USA
}

Received November 6, 1984; accepted January 28, 1985

\begin{abstract}
Summary. In this paper we discuss some of the thermodynamic and spectroscopic properties of polycyclic aromatic hydrocarbons (PAH's) which make them attractive candidates as carriers of the Diffuse Interstellar Bands (DIB's). We point out that, in the diffuse medium, many of these species will be partially hydrogenated and positively charged and will absorb in the visible. The observed lineshapes and -widths of the DIB's can be explained by rovibronic band contours and linebroadening due to internal conversion. Since little information is available concerning the spectroscopic properties of such species in the gas phase, a considerable amount of laboratory and theoretical work is needed.
\end{abstract}

Key words: interstellar medium: abundances - interstellar medium: extinction - interstellar medium: molecules - lines: identification

\section{Introduction}

The origin of more than 40 broad diffuse interstellar absorption bands (DIB's) which extend from about $4400 \AA$ into the near IR is unknown. This is not for want of trying, however, for since their interstellar origin was recognized more than 50 years ago (Merrill, 1934) an enormous effort has been made observationally, experimentally, and theoretically to understand this phenomenon. Equally strong cases can be made for a gas phase as well as a solid state origin, but neither explanation is completely adequate. Several excellent reviews of the problem can be found in the literature (Herbig, 1975; Smith et al., 1977).

Recently, Leger and Puget (1984) have suggested that polycyclic aromatic hydrocarbons (PAH's) are responsible for most of the unidentified IR emission bands which are associated with UV-rich regions of planetary and reflection nebulae, $\mathrm{H}$ in regions, stellar objects, and extragalactic sources. Allamandola et al. (1984), working independently along similar lines, attributed the emission to positively charged PAH's since, in the IR band emitting regions, the majority of these molecules will be ionized. The proposed presence of PAH's in a variety of objects points to their ubiquitous presence in the interstellar medium, an idea similar to Johnson's (1967) and Donn's (1968). In this paper, we discuss some of the thermodynamic and spectroscopic properties inherent in this class of compounds which make them attractive candidates as carriers of the diffuse interstellar bands. We also point out that, in the diffuse medium, these species will most

Send offprint requests to: L. J. Allamandola likely be present as a mixture of partially hydrogenated positive ions and neutral species which should absorb the visible. Since there is little information available concerning the spectroscopic properties of such unsaturated species in the gas phase, a considerable amount of laboratory and theoretical work is called for.

2. Polycyclic aromatic hydrocarbons: some relevant properties

\subsection{Stability and ionization potential}

If molecules are responsible for the diffuse bands, they must be unusually stable with respect to dissociation to maintain the concentration required in the harsh interstellar medium. As a class, aromatic molecules such as PAH's are much more stable than others with the same number of atoms, due to the delocalization of electrons in the molecular orbitals, and among these, there is a sub-class called super-aromatics which are even more stable (Clar, 1972). In general, for a given number of rings, the most stable PAH will have the most condensed configuration possible (Stein, 1978). We believe that it is these condensed forms which will be the most favored under interstellar conditions. For example, of the molecules shown in Fig. 1, pyrene is more stable than chrysene which is more stable than tetracene, and form A of hexabenzocoronene is much more stable than form B. Mass spectroscopic studies of ovalene and the two forms of hexabenzocoronene illustrate this: both ovalene and hex-A lose up to $5 \mathrm{H}$ atoms but suffer no loss of skeletal carbon upon bombardement by $75 \mathrm{eV}$ electrons, while hex-B readily fragments (Reed and Tennant, 1971; Bursey et al., 1970). Furthermore, these studies show that multiply charged PAH's are also quite stable.

Because of the stability of these aromatic molecules, photodissociation rates for carbon skeleton break-up are exceedingly low and the photophysics of relaxation following UV photon excitation has formed a rich area of study in itself (e.g., Birks, 1970). PAH's have low first ionization potentials, many lying in the 6-8 eV range $(\lambda<2100 \AA$, see e.g., Gallegos, 1968; Clar and Schmidt, 1977, 1978 and references therein). In general, the second ionization potential is about $20 \mathrm{eV}(620 \AA)$.

\section{2. $U V$-Vis absorption bands}

Although not specifically referring to PAH's, Platt (1956), in his pioneering work on the spectroscopy of unsaturated systems, showed that the long wavelength absorption band limit is largely determined by the dimensions of the molecule, with the longest linear dimension dominant. For example, in the series of linear 
<smiles>c1ccc2cc3cc4ccccc4cc3cc2c1</smiles>

TETRACENE $\mathrm{C}_{18} \mathrm{H}_{12}$<smiles>c1ccc2c(c1)ccc1c3ccccc3ccc21</smiles>

CHRYSENE $\mathrm{C}_{18} \mathrm{H}_{12}$<smiles>c1cc2ccc3cccc4ccc(c1)c2c34</smiles>

PYRENE $\mathrm{C}_{16} \mathrm{H}_{10}$<smiles></smiles>

OVALENE $\mathrm{C}_{32} \mathrm{H}_{14}$

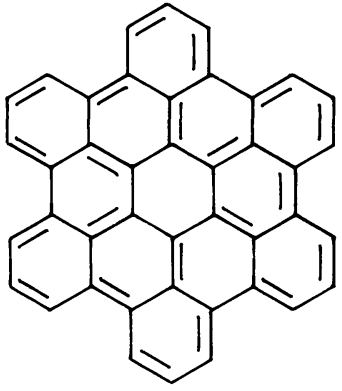

$$
\mathrm{C}_{42} \mathrm{H}_{18}
$$

HEXABENZOCORONENE A

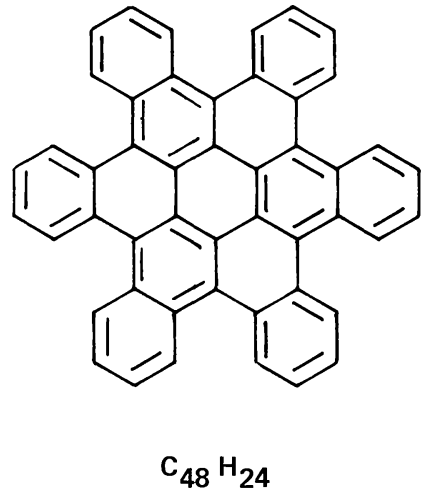

HEXABENZOCORONENE B

Fig. 1. Some polycyclic aromatic hydrocarbons. Hydrogen coverage of these molecules will be much less than shown and a large fraction of them will be positively charged in the interstellar medium

fused ring aromatics extending from naphthalene ( 2 rings) to pentacene (5 rings), the limit shifts from about 3000 to $6000 \AA$.

Similarly, for the molecules pyrene, hexabenzocoronene-A and ovalene (Fig. 1) the limits are about 3800, 4500, and $4700 \AA$, respectively (Clar, 1964). In virtually all cases the spectrum consists of several broad, largely temperature independent, overlapping bands which increase in absorption strength towards shorter wavelength, with the band near the long wavelength limit generally rather weak. In the UV, the bands generally have large molar extinction coefficients $\left(10^{4}-10^{5} 1 \mathrm{~mole}^{-1} \mathrm{~cm}^{-1}\right)$ which correspond to cross sections $\sigma$ of about $10^{-16} \mathrm{~cm}^{2}$ molecule ${ }^{-1}$ (see Birks, 1970, for a compilation of this data).

\section{Polycyclic aromatic hydrocarbons:} physical state and some spectroscopic properties expected in the interstellar medium

Together, the stability of PAH's, their low ionization potential and absorption characteristics permit one to estimate the form in which they would most likely exist in the diffuse medium. Throughout the following discussion, many assumptions have been made. Several numerical values are quite uncertain and errors as large as an order of magnitude are possible. In all cases, conservative estimates are used to deduce the physical state of the PAH's. The UV flux from 912 to $2000 \AA$ in the diffuse medium is about $10^{8} \mathrm{~cm}^{-2} \mathrm{~s}^{-1}$ (Habing, 1968). Assuming an average cross section of $10^{-16} \mathrm{~cm}^{-2} \mathrm{PAH}^{-1}$ for this wavelength range implies that each molecule would absorb a potentially ionizing photon approximately every $10^{8}$ seconds. The dominant neutralization channel should be radiative recombination. An upper limit to this rate is given by the electron-ion collision rate, $n_{e} \alpha$, where $\alpha$ is the recombination coefficient which is taken to be $610^{-7}\left(\frac{T}{300}\right)^{-0.5}$ $\mathrm{cm}^{3} \mathrm{~s}^{-1}, n_{e}$ the electron number density and $T$ the gas temperature
(Prasad and Huntress, 1980). In models of the diffuse medium, the degree of ionization is determined by the carbon content and the UV radiation field (Prasad and Huntress, 1980). Thus $n_{e} \approx 310^{-4} n_{\mathrm{H}}$. Taking $n_{\mathrm{H}}$ and $T$ equal to $10 \mathrm{~cm}^{-3}$ and $100 \mathrm{~K}$, respectively, yields a value of $310^{8}$ seconds as the time between electron-ion collisions, a value only slightly longer than the ionization period. Thus there will be comparable amounts of neutral and ionized PAH's. If the neutralization efficiency of these large molecules is lower, a predominantly ionic population of PAH's is favored.

Ionization of PAH's leaves the molecule with an unpaired electron, forming a free radical. Free radicals generally possess allowed transitions in the visible and near IR spectral regions, while their parent species, containing an even number of electrons, absorb at shorter wavelengths. For example the ${ }^{2} A^{\prime \prime}-{ }^{2} A^{\prime}$ transition of the radical HCO extends from about $4500 \AA$ to $7500 \AA$ (Herzberg and Ramsay, 1955), while the parent molecule, $\mathrm{H}_{2} \mathrm{CO}$, does not absorb strongly at wavelengths longward of $3500 \AA$ (see Herzberg, 1966, and Herzberg, 1971, for a thorough discussion of electronic absorption spectroscopy). Crawford et al. (1984) are preparing a manuscript in which the visible absorption bands of PAH cations suspended in low temperature glasses are compared with the DIB's.

An additional way in which PAH's could be present as radicals in the interstellar medium is if they are only partially hydrogenated. Each hydrogen that is removed potentially produces an additional radical "site". Support that PAH's are partially hydrogenated in at least certain regions of the interstellar medium comes from the IR emission bands. The 3.3, 6.2, 7.7, and $8.6 \mu \mathrm{m}$ bands are completely consistent with PAH's; however, that at $11.3 \mu \mathrm{m}$ is only consistent if the majority of the rings at the edge of the PAH contain no more than one $\mathrm{H}$ atom (Léger and Puget, 1984; Allamandola et al., 1984). The precise mechanism of $\mathrm{H}$ atom removal is unclear at present, but is presumably due to a combination of photolysis and electron-ion recombination reac- 
tions. The rate of the reverse process, $\mathrm{H}$ atom capture, is difficult to estimate because the geometric cross section of these radicals is unknown.

\section{Polycyclic aromatic hydrocarbons and the diffuse interstellar bands}

PAH UV absorption bands each span one to several hundred angstroms (Clar, 1964; Birks, 1970). Due to the compressed energy scale and the fact that most of the spectra have been taken of molecules in condensed phases due to experimental considerations, these bands show little structure although they are made up of many individual vibronic transitions. For the somewhat lower energy transitions which occur in the visible spectral region expected for the PAH radicals in the interstellar gas, individual rovibronic bands should be resolvable. The transitions, however, would have very small $\left(<0.01 \mathrm{~cm}^{-1}\right)$ rotational line spacings due to the large moments of inertia of PAH's (Ross, 1971; Hollas, 1973). Each vibronic transition would produce a band for which the width depends not only upon the temperature and the rotational constants, but also upon the particular transition involved. Due to the symmetry of some of these PAH's and their high degree of planarity, transitions whose principle moment lies parallel to the major symmetry axis will have rotational and vibrational selection rules which differ from those for transitions in which the moment lies perpendicular to the major symmetry axis. The cumulative effects of these constraints results in characteristic band contours (designated as $\mathrm{A}, \mathrm{B}$, or $\mathrm{C}$-type bands) which can

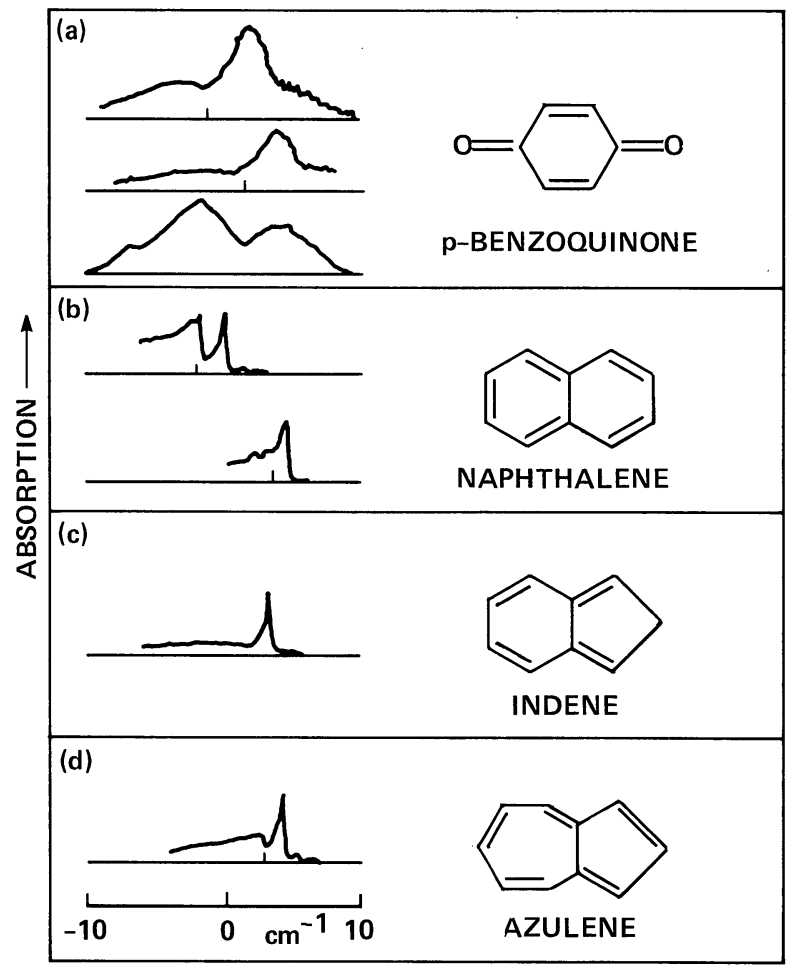

Fig. 2. Rotational band contours measured for some planar, cyclic molecules. All contours are presented on a common frequency scale which increases to the right. Actual band positions are a) p-benzoquinone-4764 and $4761 \AA$; b) naphthalene-3081 and $3123 \AA$; c) indene- $2880 \AA$; and d) azulene- $3478 \AA$. These have been reproduced with permission from Ross, Adv. Chem. Phys. 20 (1971), 341

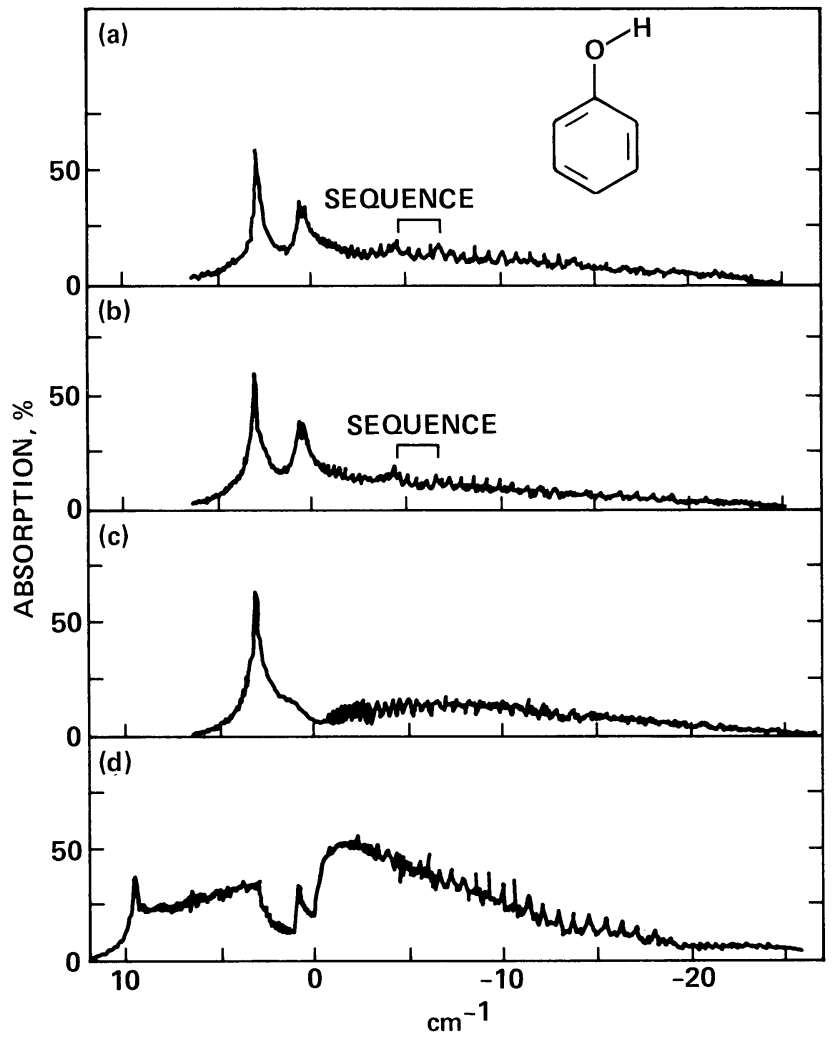

Fig. 3. Rotational band contours for the $2751 \AA$ band in phenol. Note that the frequency scale is opposite to that in Fig. 2 and decreases to the right. A) the measured band. B) Computed rotational band contour assuming it is a $B$-type band. C) Computed rotational band contour assuming it an $A$-type band. D) Computed rotational band contour assuming it is a $C$-type band. This figure has been reproduced with permission from Hollas, Molecular Spectroscopy: $a$ Specialized Periodical Report Volume 1 (1973), 63

differ in the same spectral region producing singly, doubly or multiply peaked profiles. Examples of the individual bands measured or expected from similar although smaller molecules are presented in Figs. 2 and 3.

While these molecules are not as large as the PAH's under consideration, Figs. 2 and 3 illustrate the range of possible contours vibronic transitions in large molecules can produce. These profiles are taken from the reviews of Hollas (1973) and Ross (1971). They show that the width of rovibronic bands in large molecules can vary from somewhat less than one to tens of wavenumbers.

The width of rovibronic transitions in PAH's under interstellar conditions can be roughly estimated as follows. The most populated rotational levels of a molecule have $J \approx k T /(A+C)^{1 / 2}$, in which $J$ is the total angular momentum quantum number and $A$ and $C$ are two of the rotational constants of the molecule (Ross, 1971). Assuming an equilibrium temperature $T \approx 100 \mathrm{~K}$ and rotational constants of $\approx 0.01 \mathrm{~cm}^{-1}$ of the PAH's, results in $J \approx 60$. This corresponds to linewidths similar to those in Figs. 2 and 3, in which $J \approx 40-60$. The sharper features are a few wavenumbers wide and slightly asymmetric, having a steep edge on the blue side. The asymmetry arises from the difference between the rotational constants in the ground and excited electronic state of the molecule, which causes the lower $J$ lines to run together forming a "band head" (e.g., Steinfeld, 1978). Some of the deepest DIB's are of comparable width and similarly asymmetric with the blue side steepest (Wu, 1972; Savage, 1976; Welter and Savage, 1977; Snell 
and Vanden Bout, 1981; Herbig and Soderblom, 1981; Chlewicki et al., 1985). This similarity in profile between some DIB's and rotational band contours of large molecules was previously noted by Danks and Lambert (1976).

The width of the broader DIB's may be caused by radiationless internal conversion, as was pointed out by Douglas (1977). The rate of internal conversion depends upon the interaction between the particular vibronic level excited and nearby levels of a lower electronic state, and varies greatly from one level to another. Linewidths up to $100 \mathrm{~cm}^{-1}$ are not unusual among large aromatic molecules (Byrne and Ross, 1971). It is worth noting that the broader DIB-features tend to lie at shorter wavelengths (Herbig, 1975), which is consistent with internal conversion because the density of states of molecules generally increases with energy.

Although correlation studies among the DIB's are hampered by the weak nature of most of the features, there are strong indications that some of them do not correlate (Chlewicki et al., 1985; Baines and Whittet, 1983; Snow, 1973; Savage, 1976). It is interesting to speculate by creating a vibrational energy level diagram with spacings corresponding to the energies of the IR emission bands at $6.2,7.7$, and $8.6 \mu \mathrm{m}$, assigned to the in-plane fundamental vibrations in PAH's (Cyvin et al., 1982). In these molecules the vibrational frequencies in the ground and upper electronic states will be similar (Amirav et al., 1980). Although the separation between a number of DIB's can be matched by the spacings mentioned above there does not seem to be a consistent way in which the bands can be fitted into a single energy level diagram. However, in a mixture of PAH's the spectrum may be very complex, because of the large and varying number of vibronic transitions which may be allowed in each molecule. Alternatively, since the number of diffuse lines is large, the apparent agreement in spacing with that between some of the IR bands may just be fortuitous.

The fraction of available carbon required by these molecules to account for the DIB's can be estimated as follows: the column density of absorbers can be calculated with the well-known formula

$W_{\lambda}=\frac{\pi e^{2}}{m c^{2}} \lambda_{0}^{2} N f$

where $W_{\lambda}$ is the equivalent width of a particular line, $e^{2} / m c^{2}$ the classical radius of the electron, $\lambda_{0}$ the central wavelength of the band, $N$ the column density of absorbers and $f$ the oscillator strength. Herbig (1975) has measured an equivalent width of $805 \mathrm{~m} \AA$ for the $5780 \AA$ band toward Cyg OB2 \# $5\left(=+40^{\circ} 4220\right)$ for which $E(B-V)$ is 2.02 (see also Chlewicki et al., 1985). For an allowed electronic transition, $f$-values range from 0.1 to 1.0. Taking an $f$-value of 0.1 results in a column density of absorbers equal to $2.810^{13} \mathrm{~cm}^{-2}$. The column density of hydrogen towards Cyg OB2 \# 5 is calculated using $N_{\mathrm{H}} / E(B-V)=4.210^{21} \mathrm{~cm}^{-2}$ $\mathrm{mag}^{-1}$, to be $8.510^{21} \mathrm{~cm}^{-2}$ (see, for example, Greenberg, 1978). Since the cosmic abundance of carbon relative to hydrogen is about $3.710^{-4}$, the column density of carbon is $3.110^{18} \mathrm{~cm}^{-2}$ along this line of sight. Considering PAH's composed of $30 \mathrm{C}$ atoms as typical of the diffuse band carriers, this requires $8.310^{14}$ carbon atoms. This represents less than 0.03 percent of the available carbon.

\section{Polycyclic aromatic hydrocarbons, the FUV extinction, and the $2200 \AA$ hump}

Most of the correlation studies between the DIB's and the FUV extinction or the $2200 \AA$ hump have been restricted to the $4430 \AA$ band (Wu et al., 1981; Nandy et al., 1982; Witt et al., 1983; Seab and Snow, 1984). The observations show a weak correlation between the $4430 \AA$ band and the $2200 \AA$ hump and no correlation with FUV extinction. In addition, Seab and Snow (1984) report no correlation between the 5780 and $6284 \AA$ band with both the FUV extinction and $2200 \AA$ hump. Since large organic molecules such as PAH's generally show stronger absorption bands in the UV than in the visible, it is interesting to estimate the contribution they could make to the FUV extinction. The ratio of the optical depth in the FUV $\left(\tau_{\text {fuv }}\right)$ to that in the visible $\left(\tau_{\text {vis }}\right)$ can be obtained from:

$\frac{\tau_{\text {fuv }}}{\tau_{\text {vis }}}=\frac{f_{\text {fuv }}}{f_{\text {vis }}} \cdot \frac{\Delta v_{\text {vis }}}{\Delta v_{\text {fuv }}}$.

Consider an electronic transition at $1250 \AA$, with a typical PAH width of $100 \AA\left(\Delta v \approx 6000 \mathrm{~cm}^{-1}\right)$, and a visible transition with $\Delta v=8 \mathrm{~cm}^{-1}$ (the width of the $5780 \AA$ band). For a star with $E(B-V)$ $=1.5$, the detection limit is about $\tau_{\text {fuv }}=0.1$, depending on the width of the transition and the wavelength region where it absorbs (Chlewicki, private communication). For this amount of reddening, the $5780 \AA$ band has a $\tau_{\text {vis }}$ of about 0.4 (see the example of Cyg OB2 \# 5 in the previous section). Substituting these numbers, one finds that for $\frac{f_{\text {fuv }}}{f_{\text {vis }}} \leqq 200$, the electronic transition in the FUV would be undetectable with the present quality of IUE data. Concerning the $2200 \AA$ hump, experimental studies have shown that laboratory synthesized amorphous carbon particles, which are presumably made up of a collection of PAH-like molecules, exhibit an extinction peak between 2000 and $3000 \AA$ A. Borghesi et al. (1984), in summarizing this work, show that the peak shifts towards shorter wavelengths for the smaller sized particles and speculate that for particles less than $40 \AA$ in radius, the band would peak at about $2200 \AA$. The low abundance of PAH's required to account for the DIB's suggests that the species which give(s) rise to the $2200 \AA$ hump are (is) of a different nature unless the oscillator strength in the visible is much less and these molecules absorb very strongly in the UV near $2200 \AA$. Of course, this does not rule out the possibility that larger molecules (small particles?), which do not contribute to the DIB's could contribute to the $2200 \AA$ hump. In this respect, it is interesting to note that in 1968 Donn suggested that although the individual spectra did not match, a collection of PAH's might account for the $2200 \AA$ hump.

\section{Future work}

Based on the above, we believe that PAH radicals and radical ions are possible constituents of the interstellar medium and that absorption by these species may be responsible for the diffuse interstellar bands. At this point we would like to make some suggestions for future research in order to test this hypothesis. 1) More observations involving correlation studies are needed to determine which DIB's arise from the same carrier, followed by a vibrational analysis. Furthermore, correlation studies between the DIB's and the FUV extinction or $2200 \AA$ hump need to be extended. 2) In addition to observations, laboratory and theoretical studies of the types of molecules, radicals and radical ions proposed here are essential to establish their spectroscopic properties and to calculate the band contours of the different types of rovibronic transitions possible in these species.

In the course of preparing this manuscript we learned that A. Leger and L. B. d'Hendecourt were pursuing a similar line of reasoning and preparing the paper which appears adjacent to this. 
Acknowledgements. L.J.A. wishes to thank Xander Tielens and John Barker for many helpful discussions concerning the properties of PAH's under interstellar conditions. G.v.d.Z. thanks Grzegorz Chlewicki, Leo van Ijzendoorn, Fred Baas, and Mayo Greenberg for stimulating discussions during the preparation of this manuscript, and gratefully acknowledges the support of the Netherland's "Stichting voor Fundamenteel Onderzoek der Materie (F.O.M.)". Furthermore, we acknowledge critical reading of the manuscript by Gregory Seab.

\section{References}

Allamandola, L.J., Tielens, A.G.G.M., Barker, J.R.: 1984, Astrophys. J. Letters (in press)

Amirav, A., Even, U., Jortner, J.: 1980, Opt. Comm. 32, 266

Baines, D.W.T., Whittet, D.C.B.: 1983, Monthly Notices Roy. Astron. Soc. 203, 419

Birks, J.B.: 1970, Photophysics of Aromatic Molecules, John Wiley and Sons, Ltd., London

Borghesi, A., Bussoletti, E., Colangelli, L.: 1984, Astron. Astrophys. (in press)

Bursey, M.M., Rogerson, P.F., Bursey, J.M.: 1970, Org. Mass. Spectrom. 4, 615

Byrne, J.P., Ross, I.G.: 1971, Australian J. Chem. 24, 1107

Chlewicki, G., van der Zwet, G.P., van Ijzendoorn, L.J., Greenberg, J.M., Alvarez, P.P.: 1985, Astrophys. J. (submitted)

Clar, E.: 1964, Polycyclic Hydrocarbons, Academic Press, London

Clar, E.: 1972, The Aromatic Sextet, John Wiley and Sons, London

Clar, E., Schmidt, W.: 1977, Tetrahedron 33, 2093

Clar, E., Schmidt, W.: 1978, Tetrahedron 34, 3219

Crawford, M., Tielens, A.G.G.M., Allamandola, L.J.: 1984, Astrophys. J. Letters (in preparation)

Cyvin, S.J., Cyvin, B.N., Brunvoll, J., Whitmer, J.C., Klaeboe, P.: 1982, Z. Naturforsch. 37a, 1359 and references therein

Danks, A.C., Lambert, D.L.: 1976, Monthly Notices Roy. Astron. Soc. 174, 571

Donn, B.: 1968, Astrophys. J. 152, L129
Douglas, A.E.: 1977, Nature 269, 130

Gallegos, E.J.: 1968, J. Phys. Chem. 72, 3452

Greenberg, J.M.: 1978, Interstellar Dust in Cosmic Dust, Chapter 4, ed. J. A. M. McDonnell, J. Wiley and Sons, Ltd., 187

Habing, H.: 1968, Bull. Astron. Inst. Ned. 19, 421

Herbig, G.H.: 1975, Astrophys. J. 196, 129

Herbig, G.H., Soderblom, D.R.: 1982, Astrophys. J. 252, 610

Herzberg, G., Ramsay, D.A.: 1955, Proc. Roy. Soc. A 3, 34

Herzberg, G.: 1966, Molecular Spectra and Molecular Structure III, Van Nostrand and Reinhold Co., New York

Herzberg, G.: 1971, The Spectra and Structure of Simple Free Radicals, Cornell Univ. Press, Ithaca

Hollas, J.M.: 1973, Chapter 2 in Molecular Spectroscopy: A Specialized Periodical Report Volume 1, The Chemical Society, London, 63

Johnson, F.M.: 1967, in Interstellar Grains, ed. J.M. Greenberg and T.P. Roark, NASA-SP-140, 229

Leger, A., Puget, J.L.: 1984, Astron. Astrophys. 137, L5

Merrill, P.W.: 1934, Publ. Astron. Soc. Pac. 46, 206

Nandy, K., Morgan, D.H., Houziaux, L.: 1982, Astrophys. Space Sci. 85, 159

Platt, J.R.: 1956, Astrophys. J. 123, 486

Prasad, S.S., Huntress, W.T., Jr.: 1980, Astrophys. J. Suppl. Ser. 43, 1 (see p. 5 for discussion of $\alpha$ )

Reed, R.I., Tennent, A.: 1971, Org. Mass. Spectrom. 5, 619

Ross, I.G.: 1971, Adv. Chem. Phys. 20, 341

Savage, B.D.: 1976, Astrophys. J. 205, 122

Seab, C.G., Snow, T.P.: 1984, Astrophys. J. 277, 200

Smith, W.H., Snow, T.P., York, D.G.: 1977, Astrophys. J. 218, 124

Snell, R.L., Vanden Bout, P.A.: 1981, Astrophys. J. 244, 844

Snow, T.P.: 1973, Astron. J. 78, 913

Stein, S.E.: 1978, J. Phys. Chem. 82, 566

Steinfeld, J.I.: 1978, Molecules and Radiation, MIT Press, Cambridge, Mass., p. 127-128

Welter, G.L., Savage, B.D.: 1977, Astrophys. J. 215, 788

Witt, A.N., Bohlin, R.C., Stecher, T.P.: 1983, Astrophys. J. 267, L47

Wu, C.: 1972, Astrophys. J. 178, 681

Wu, C., York, D.G., Snow, T.P.: 1981, Astron. J. 86, 755 